\title{
Secondary Structure Prediction of RNA using Machine Learning Method
}

\author{
Romasa Qasim \\ Department of Computer \\ Science, \\ University of Karachi, \\ University Road, Karachi, 75270, \\ Pakistan
}

\author{
Nishat Kauser \\ Department of Computer \\ Science, \\ University of Karachi, \\ University Road, Karachi, 75270, \\ Pakistan
}

\author{
Dr. Tahseen Jilani \\ Department of Computer \\ Science, \\ University of Karachi, \\ University Road, Karachi, 75270, \\ Pakistan
}

\begin{abstract}
Ribonucleic Acid (RNA) plays a vital role in the transcription process. Since the information stored in DNA is converted into sequences of a chemical compounds named amino acids through mRNA in order to produce the ultimate gene product i.e., protein. The importance of RNA in the transcription process gives a better justification to analyze it. RNA cannot exist stably in its primary structure, thus, to attain a stable structure, it folds back on itself to form secondary structure (2o RNA) and further folding of RNA nucleotides gives rise to the tertiary structure (3o RNA). In this paper, a new model using neural network for RNA secondary structure prediction is proposed. Our computational model predicts multiple secondary structures of a single RNA by applying a parallel algorithm for finding near maximum independent set in the circle graph proposed by Takefuji Y. et al (1990). Based on frequency density analysis of the predicted RNA secondary structures, we proposed an optimized secondary structure of RNA among all the possibilities using statistical probability distributions. The paper concludes by discussing the nature and behavior of 2o RNA predicted by our method and a comparison with the results of other researchers. We have shown that the proposed model has better accuracy as compared to the other researches.
\end{abstract}

\section{General Terms}

Machine Learning, Neural Network, Bioinformatics

\section{Keywords}

Ribonucleic Acid, RNA, Neural Network, machine learning

\section{INTRODUCTION}

RNA molecules are integral components of the cellular machinery for protein synthesis and transport, transcriptional regulation, chromosome replication, RNA processing and modification, and other fundamental biological functions [1][2][3]. This macromolecule is basically composed of four fundamental molecules i.e., Adenine (A), Cytosine (C), Gaunine (G) and Uracil (U). The molecules are same as that of DNA accept Uracil. DNA has Thymine (T) instead of Uracil (U). Another structural difference is that DNA is double stranded, however in most cases, RNA is single stranded. In the presence of salty water, RNA forms intrastrand base-pairs, which result in the formation of secondary structure. Under appropriate conditions, the secondary structure folds back around itself to form tertiary structure of
RNA. This folding process usually depends on the presence of divalent ions like magnesium ions and on the temperature [4]. Most of the time, the structure of an RNA is very important in biological processes. Also a particular level of structure may be helpful in determining the possible next structure. For example, the secondary structure of RNA can be used to explain translational controls in mRNA [5][6] and replication controls in single-stranded RNA viruses [7]

The base-pairs which take part in the formation of secondary structure of RNA are the Watson-Crick complementary bases i.e., $\mathrm{C}-\mathrm{G}$ and $\mathrm{A}-\mathrm{U}$ and Wobble base pair i.e., G-U, which occurs occasionally.

A primary physical method to determine the secondary structure of RNA is through X-ray crystallography in which pure sample of RNA molecules are crystallized and then X-rayed, the data is collected on resulting X-ray diffraction. After this, electron density model is prepared and refined progressively in order to achieve secondary structure of that RNA sample. Due to its difficulty and slow speed it is not possible to perform this expensive procedure for several times with different samples of RNA, therefore, many alternate methods are used to predict the secondary structure of RNA. Since this method is quite complex and time consuming, attention diverted toward the computerbased simulation of secondary structure of RNA.

Until now, much progress has been made in the computational simulation of RNA secondary structure prediction. Dynamic programming is one of the old and widely accepted techniques. This method of secondary structure prediction was first proposed by waterman [8], Waterman and Smith [9] and Nussinov [10]. The drawback of this method of prediction is its computational time. The behavior of dynamic programming algorithms is found to be of $\mathrm{O}\left(\mathrm{n}^{4}\right)$, which is too slow to be effective, for bigger sequences in particular, since the behavior is exponential. Several attempts to modify the dynamic programming algorithms have been made and are considered to be successful. Another method of determining the secondary structure of RNA is the comparative method, which works simultaneously with more than one sequence in order to find an identical structure. Sankoff [11] extended the dynamic programming approach by folding and aligning multiple sequences to generate a phylogenetic tree for secondary structure prediction. But the technique proposed by Sankoff couldn't proven itself to be excellent with more than a couple of sequences. The Zuker algorithm, implemented in the 
programs MFOLD [12] and ViennaRNA [13], is an efficient dynamic programming algorithm for identifying the globally minimal energy structure for a sequence, as defined by such a thermodynamic model [11][14][15]. The Zuker algorithm requires $\mathrm{O}\left(\mathrm{N}^{3}\right)$ time and $\mathrm{O}\left(\mathrm{N}^{2}\right)$ space for a sequence of length $\mathrm{N}$.

Another paradigm is the use of artificial intelligence to predict the structure. Takefuji took the advantage of ANN's parallel approach to converge toward an optimal solution by applying NN to predict the near maximum independent set of circle graph within several hundred iterations in his paper [16] and used nearmaximum independent set of circle graph to find the optimal structure of single stranded RNA. This work is further improved in [17] with the use of Hopfield Neural Network and by proving the technique by presenting a good comparison between other techniques and the proposed technique. A similar technique was proposed by Steeg [18] and he used Hopfield neural network, Boltzman Machine and Mean field Theory (MFT) network and presented results using circle graph.

We modify the technique presented by Takefuji and we predict the optimal and sub-optimal secondary structures of single stranded RNA from its primary structure by using our new machine learning method. We use different probability distributions to converge toward the solution and discuss the behavior and role of these distributions in order to predict the secondary structure of RNA and propose a distribution which may help to converge to the solution most optimally.

\section{STATISTICAL REVIEW}

Nearly all of the real-world problems have some means of randomness in it. Likewise in human machinery there may present many sources of randomness. For example, there is no fix figure that how much cells a human body produce in a day. Most of the time these random figures follow a particular pattern so, this randomness is normally represented by a probability distributions. Probability distribution function is one which represents all possible values which a random variable can take, provided with a range of minimum and maximum values.

Takefuji suggested in paper [16] to use small negative numbers in the start of simulati. Following the same, we only use those distributions which can vary in positive as well as negative dimension. So, in our NN simulator we use five distribution techniques and further analyze the behavior of results. Following is a brief description of these probability distributions [19].

a). Uniform distribution: it is a special case of beta distribution in which $\alpha_{1}=\alpha_{2}=1$. It has a density function:

$$
f(x)=\left\{\begin{array}{cc}
\frac{1}{b-a} & \text { if } a \leq x \leq b \\
0 & \text { otherwise }
\end{array}\right.
$$

Where $a, b$ belongs to real numbers, $a$ must be less than $b, a$ is a location parameter and $b-a$ is the shape parameter. Both the parameters are so selected that results in a negative number, which is the requirement of our particular scenario. Fig 1(a) shows general behavior of Uniform distribution.

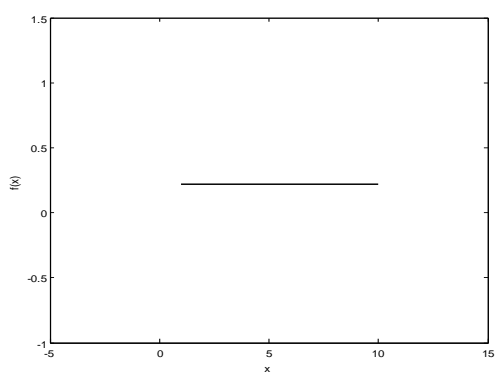

Figure 1(b) Uniform Distribution; U(0, 1) density function

b). Normal Distribution: It is the most commonly used distribution. It is the system of random variables in which all the values of variables are distributed normally with mean $\mu$ and variance $\sigma$. Normal distribution can be represented as:

$$
f(x)=\frac{1}{\sqrt{2 \pi \sigma^{2}}} e^{-\frac{(x-\mu)^{2}}{2 \sigma^{2}}} \quad \text { for all real number } x
$$

where, $\mu$ is the location parameter and $\sigma$ is the scale parameter. In our scenario we generated $\mathrm{X} \sim \mathrm{N}\left(\mu, \sigma^{2}\right)$, in such a case $\mathrm{e}^{\mathrm{x}}$ has the lognormal distribution with parameter $\mu$ and $\sigma$ denoted as LN $\left(\mu, \sigma^{2}\right)$. We have selected both the parameters such that the resulting normal random variable will be a negative number.

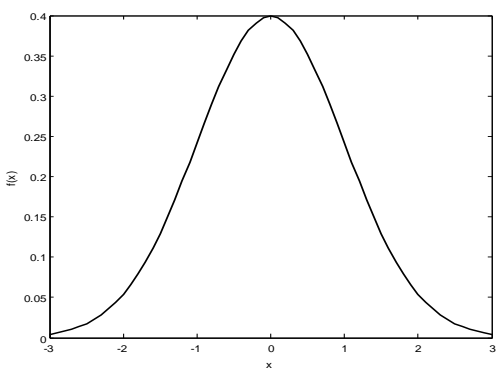

Figure 1(b). Normal Distribution; $N(0,1)$ density function

c). Johnson System: Johnson [20] proposed method for generating distributions of random variable $X$ supported by $Q=(0, \infty)$ and $Q$ $=(0,1)$ by means of transformation $\varphi: \mathrm{R} \rightarrow \mathrm{Q}$ defined by:

$\begin{array}{ll}\mathrm{Y}=\varphi^{-1}(X)=\log X & \text { if } \mathrm{Q}=(0, \infty) \\ \mathrm{Y}=\varphi^{-1}(X)=\log \frac{X}{(1-X)} & \text { if } \mathrm{Q}=(0,1),\end{array}$

Where $\mathrm{Y}$ is the prototype supported by R. For our particular scenario, we used Johnson System which is based on the transformations of normal random variable. The cases we used are SU and SB corresponding to Logistic and hyperbolic sine transformation respectively. Both the transformations can be written in general as:

$$
X=\gamma+\delta . \Gamma\left(\frac{Z \xi}{\lambda}\right)
$$

Where $\mathrm{Z} \sim \mathrm{N}(0,1)$ and $\Gamma$ is the transformation, all other parameters are scale and location parameters.

The JSB probability density function has the form of: 


$$
f(x)=\left\{\begin{array}{cc}
\frac{\alpha_{2}(b-a)}{(x-a)(b-x) \sqrt{2 \pi}} e^{-\frac{1}{2}\left[\alpha_{1}+\alpha_{2} \ln \left(\frac{x-a}{b-x}\right)\right]^{2}} & \text { if } a<x<b \\
0 & \text { otherwise }
\end{array}\right.
$$

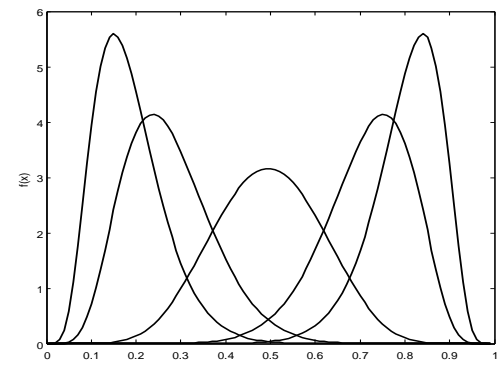

Figure 1(c). Johnson Bounded Distribution; $\operatorname{JSB}\left(\alpha_{1}, \alpha_{2}, 1\right)$ density function

Thus the JSB probability density function has the domain of $\mathrm{X}$ lying between $a$ and $b$, fig 1(c). In the prediction of secondary structure of RNA, we have assumed $a, b, \alpha_{1}$ and $\alpha_{2}$ as randomly selected negative variables, so that the resulting random variable $\mathrm{U}$ will be a negative number. Every $X \sim \operatorname{JSB}(\alpha 1, \alpha 2, a, b)$ if and only if:

$$
\alpha_{1}+\alpha_{2} \ln \left(\frac{X-a}{b-X}\right) \sim N(0,1)
$$

The other transformation of Johnson System, that is, the logistic transformation has the form:

$$
f(x)=\frac{\alpha_{2}}{\sqrt{2 \pi} \sqrt{(x-y)^{2}+\beta^{2}}} e^{-\frac{1}{2}\left\{\alpha_{1}+\alpha_{2} \ln \left[\frac{x-\gamma}{\beta}+\sqrt{\left(\frac{x-\gamma}{\beta}\right)^{2}+1}\right]\right\}^{2}} ;
$$$$
\text { for } \infty<x<\infty
$$

Where $\gamma$ is the location parameter

$$
\begin{aligned}
& \beta \text { is the scale parameter and } \\
& \alpha \text { is the shape parameter }
\end{aligned}
$$

In Johnson System logistic transformation of normal random variable the domain of X may vary between $-\infty$ to $+\infty$. We choose arbitrary values of location, shape and scale parameter which yields negative value.

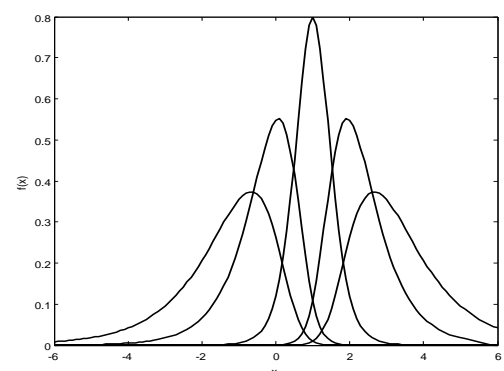

Figure 1(d). Johnson Unbounded Distribution; JSU( $\alpha_{1}, \alpha_{2}, \beta$, $\gamma$ ) density function
To be eligible to be called a JSU random variable, it should have the following form:

$$
\alpha_{1}+\alpha_{2} \ln \left[\frac{X-\gamma}{\beta}+\sqrt{\left(\frac{X-\gamma}{\beta}\right)^{2}+1}\right] \sim N(0,1)
$$

The behavior of Johnson Unbounded distribution is shown in fig 1 (d) by varying the values of $\alpha_{1}$ and $\alpha_{2}$, and keeping the values of $\beta$ and $\gamma$ fixed.

d). Triangle Distribution: A triangle distribution has lower limit of $a$ and upper limit of $b$, with mode $c$. Its probability density function is:

$$
f(x)=\left\{\begin{array}{cc}
\frac{2(x-a)}{(b-a)(c-a)} & \text { if } a \leq x \leq c \\
\frac{2(b-x)}{(b-a)(b-c)} & \text { if } c<x \leq b \\
0 & \text { otherwise }
\end{array}\right.
$$

In triangle distribution, $\mathrm{x}$ starts from a, then it get peaked at $\mathrm{c}$, which is the mode and finally reached its upper limit, that is, b. In order to generate $\mathrm{X}$ that belongs to triangle distribution, it must satisfy the following:

$$
X=\left\{\begin{array}{cl}
\sqrt{c u} & \text { if } 0 \leq u \leq c \\
1-\sqrt{(1-c)(1-u)} & \text { if } c<u \leq 1
\end{array}\right.
$$

where $\mathrm{u} \sim \mathrm{U}(0,1)$ and $\mathrm{c}$ is the mode, which is an arbitrary chosen value in our case.

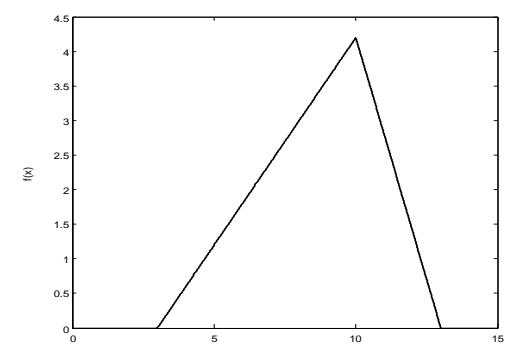

Figure 1(e). Triangle Distribution; triang(a, b, c) density function

The Triangle distribution, fig 1(e), offers considerable flexibility in its shape, coupled with the intuitive nature of its defining parameters and speed of use.

\section{METHODOLOGY}

We used a new machine learning method to predict the secondary structure of RNA. Our algorithm works in three levels. In first level, our proposed method finds all possible secondary structures from its primary sequence for all distributions discussed above. In second level, we refined the results by selecting an optimal structure from the bulk of structures achieved as an output of each distribution from the previous level. Finally, from these optimal structures of different distributions, a final structure was obtained using some statistical techniques in order to conclude the work. The optimal structure proposed in this stage may or may not be similar to the structures found in the previous level. 
We extended the work of Takefuji [16] with little modifications in the algorithm. Beside the algorithm, we also change the type of neural network that has been used by Qi Liu. He used Hopfield nets in his paper [17]. Hopfield nets are recurrent neural networks which has scalar value associated with each state of the network. This scalar value is termed as 'energy' (E), which can mathematically be defined as:

$$
E=-\frac{1}{2} \sum_{i, j} w_{i, j} s_{i} s_{j}+\sum_{i} \theta_{i} s_{i}
$$

where,

$\mathrm{w}_{\mathrm{ij}}=$ weight connecting unit $i$ with unit $j$

$\mathrm{s}_{\mathrm{i}}=$ state of the unit $i$

$\theta_{\mathrm{i}}=$ threshold of unit $i$

We haven't use Recurrent or Back-propagations Networks in order to avoid the complexity and because we didn't consider any learning process. Instead of Hopfield neural network, we customized a new model of Neural Network to predict the structure, shown in fig 2. Our model is based on the Kolgomorov's theorem, which describes the non-linear function approximator mathematically as:

$$
y\left(x_{1}, x_{2}, \ldots, x_{n}\right)=\sum_{i=1}^{2 n+1} g_{i}\left(\sum_{j=1}^{n} h_{j i}\left(x_{i}\right)\right)
$$

where $h_{j i}=$ nonlinear monovariable functions and $y=$ continuous nonlinear real function

In equation (1) if $h_{j i}=w_{i j}+b_{i}$, where $w_{i j}$ is the weight associated from one unit to another unit and $b_{i}$ is the bias associated with hidden layer. Let $M=2 n+1$ so, Kolgomorov's theorem can be modified to design a neural network as follows:

$$
y=f\left(x_{1}, x_{2}, \ldots ., x_{n}\right)=\sum_{i=1}^{M} g_{i}\left(\sum_{j=1}^{M}\left(w_{i j}+b\right) . x_{i}\right)
$$

The equation above shows the network of $\mathrm{n}$ input nodes, which represents the input layer. $W_{i j}+b_{i}$ is the summation of weights coming from the units of input to the units of hidden layer and the bias associated with the hidden layer which contains M nodes. At the end, output node is the combination of the results of hidden nodes. This kind of neural network [21] is shown in fig 2.

We use $n$ neurons in the hidden layer where $n$ is the number of base-pairs possible in an RNA sequence, considering only Watson-Crick base-pairs i.e., A-U and C-G. We avoided Wobble base-pair in our paper; however, the system has the capabilities to consider this base-pair as well.

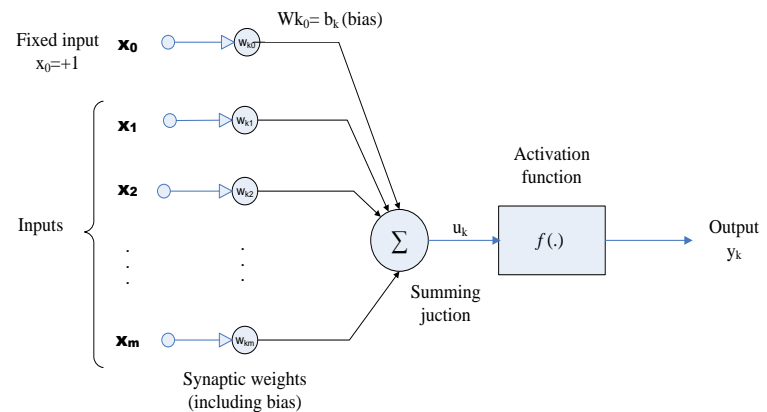

Figure 2. Proposed architecture of Artificial Neural Network (ANN) based on Kolgomorov's theorem

The procedure we followed in the parallel algorithm is as follows:

1) First we find all possible base-pairs of the RNA sequence

2) Set iterator $t=0$

3) Initialize random variable $U_{i}(t)$, where $i=1,2, \ldots n$, with small negative numbers using continuous probability distribution.

4) Evaluate values of $V_{i}(t)$, where $i=1,2, \ldots, n$ using the following binary function

$$
V_{i}(t)=\left\{\begin{array}{rr}
1 & \text { if } U_{i}(t)>0 \\
0 & \text { otherwise }
\end{array}\right.
$$

5) Compute the value of $\Delta U_{i}(t)$ using motion equation:

6) Increment $t$ by 1 till 8000

$$
\begin{gathered}
\Delta U_{i}(t)=A\left(\sum_{j=1}^{n} \operatorname{dij}\left(1-V_{j}(t)\right)(\operatorname{distance}(i))^{-1}\right) \\
.\left(1-V_{i}(t)\right)-B h\left(\sum_{j=1}^{n} \operatorname{dij}\left(1-V_{j}(t)\right)\right) V_{i}(t)
\end{gathered}
$$

We used different probability distributions to initialize $U_{i}(t)$ in step 2 and analyzed the behavior of system. We use different samples of RNA sequences and tested these sequences in our system.

\section{RESULTS AND DISCUSSION}

The different distribution techniques we applied in the algorithm mentioned above show different behavior. With a number of sequences and five different distribution techniques for each sequence, we observed that on eight thousand epochs per distribution, the results of random variables generated by the hyperbolic transformation of normal random variable with its identity transformation is the best as compared to other distributions.

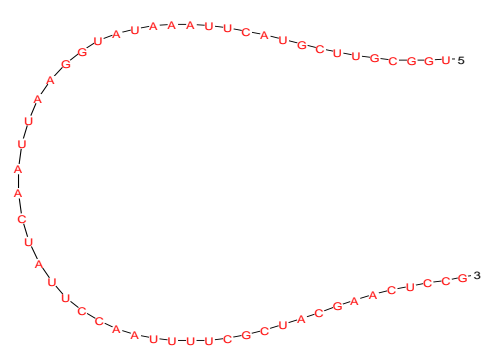

Figure 3(a). R17 viral RNA 


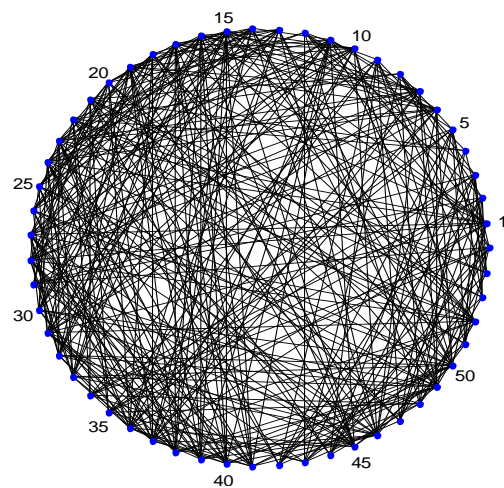

Figure 3(b). All possible Watson-Crick base-pairs for a RNA sequence of R17 viral RNA

We mention here a sequence of 55 bases from R17 viral RNA, fig 3(a). We first determine all possible base-pairing of this sequence by considering Watson-Crick base-pair only which is shown in fig 3(b). Our algorithm detects 360 possible base-pairs for this sequence of 55 nucleotides by following the rule of Tinoco in his paper [22] in which he mentioned that hairpin loop with less than three bases is sterically unlikely to occur. In the first level of our method, the system find out all eligible secondary structures from the one shown in fig 3(b).

Table 1 presents the number of proposed secondary structures by each distribution technique for the sequence of R17 viral RNA. The statistics shown in table 1 ignores all repetitions of possible secondary structures and only mutually exclusive structures are considered. We precede the analysis by finding the optimal secondary structure by statistically analyzing all eligible secondary structures.

Table 1. Number of secondary structures proposed by the system using different distribution techniques

\begin{tabular}{|l|r|}
\hline $\begin{array}{c}\text { Probability Distribution } \\
\text { Function (PDF) }\end{array}$ & $\begin{array}{c}\text { Number of Proposed } \\
\text { Secondary Structure }\end{array}$ \\
\hline Uniform & 16 \\
\hline Normal & 83 \\
\hline Johnson Bounded & 228 \\
\hline Johnson Unbounded & 252 \\
\hline Triangle & 238 \\
\hline
\end{tabular}

The correlation coefficient of each structure, proposed by the system, is measured on the basis of a previously known structure. By considering the structure predicted by Tinoco [22] for the same R17 viral RNA, we calculated the correlation coefficient by using follwing formula [17]:

$\mathrm{CC}(\%)=\sqrt{\frac{\mathrm{TP}}{\mathrm{TP}+\mathrm{FN}} * \frac{\mathrm{TP}}{\mathrm{TP}+\mathrm{FP}}} \times 100 ; \quad 0<\mathrm{CC}<100$

Where, $\mathbf{T P}=$ Base pairs that area predicted and exists in the known structure, $\mathbf{F P}=$ Base pairs that are predicted but do not exist in the known structure, $\mathbf{F N}=$ Base pairs that are not predicted but exist in the known structure, respectively.
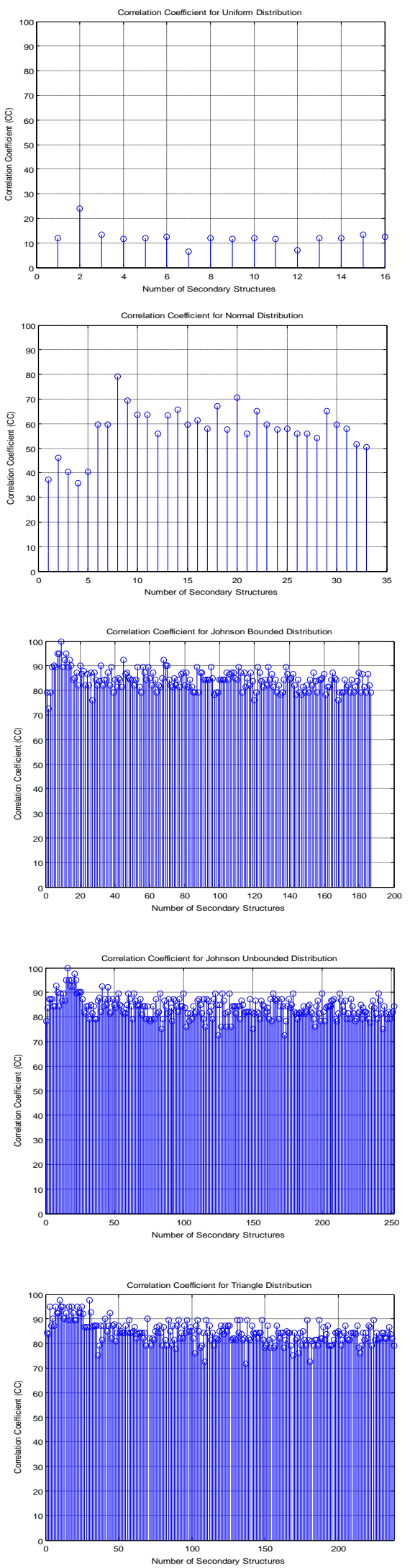

Figure 4. Correlation Coefficients of all distributions 
$\mathrm{CC}$ can be defined as the sensitivity of its geometric mean. Higher CC means better prediction accuracy. CC of all distribution techniques are shown in figure 4.

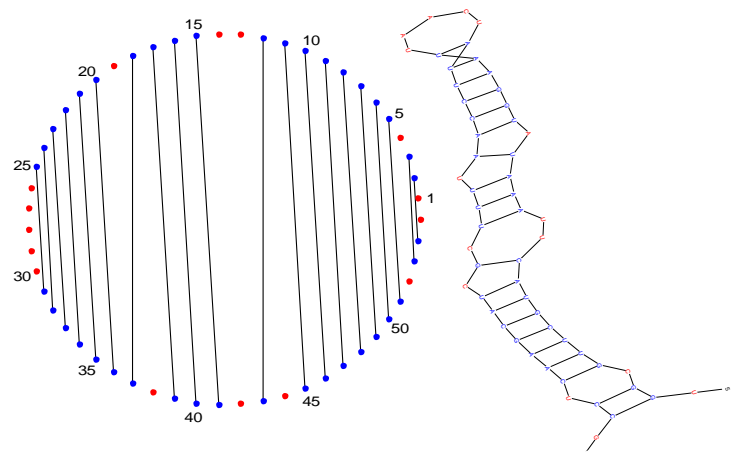

Figure 5. Circle and Sequence diagram of proposed secondary structure by our machine learning algorithm

The machine learning method predicted the possible secondary structure of this RNA sequence, figure 5, which is same as that of Tinoco's [22] prediction.
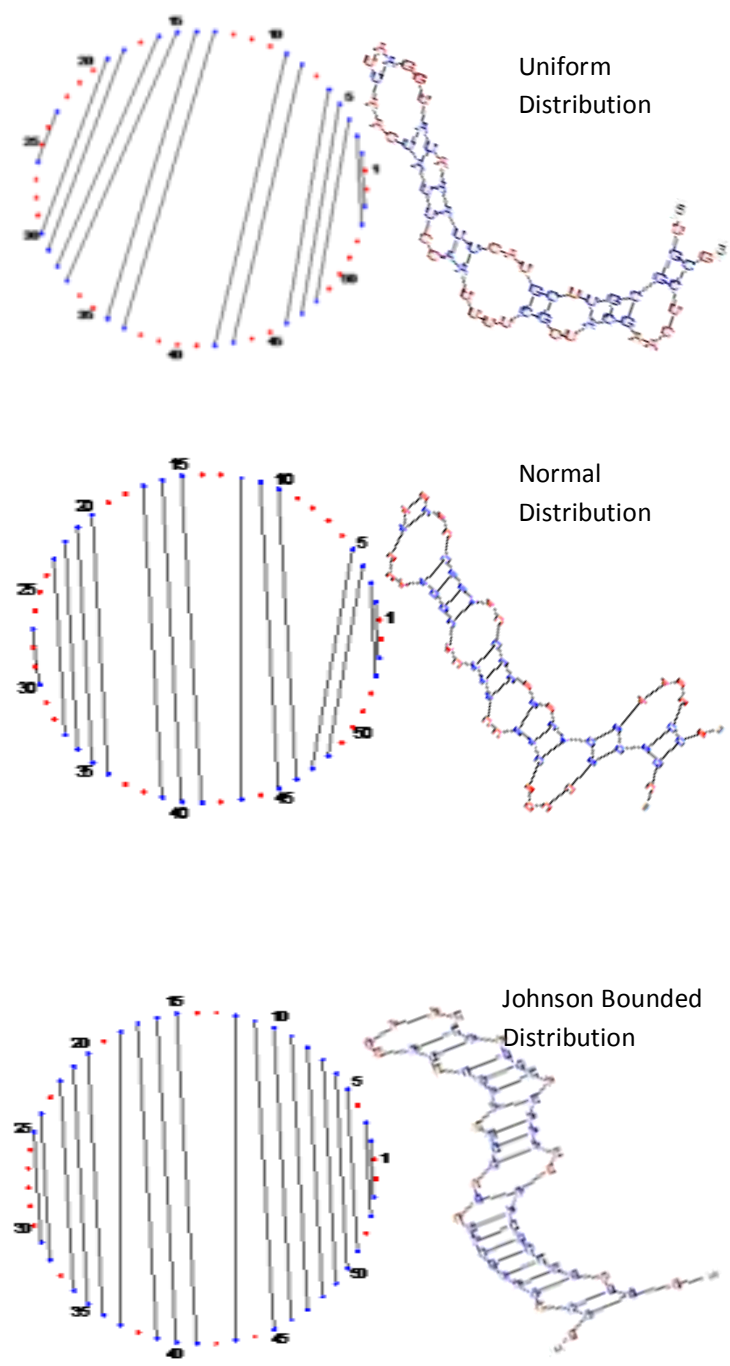
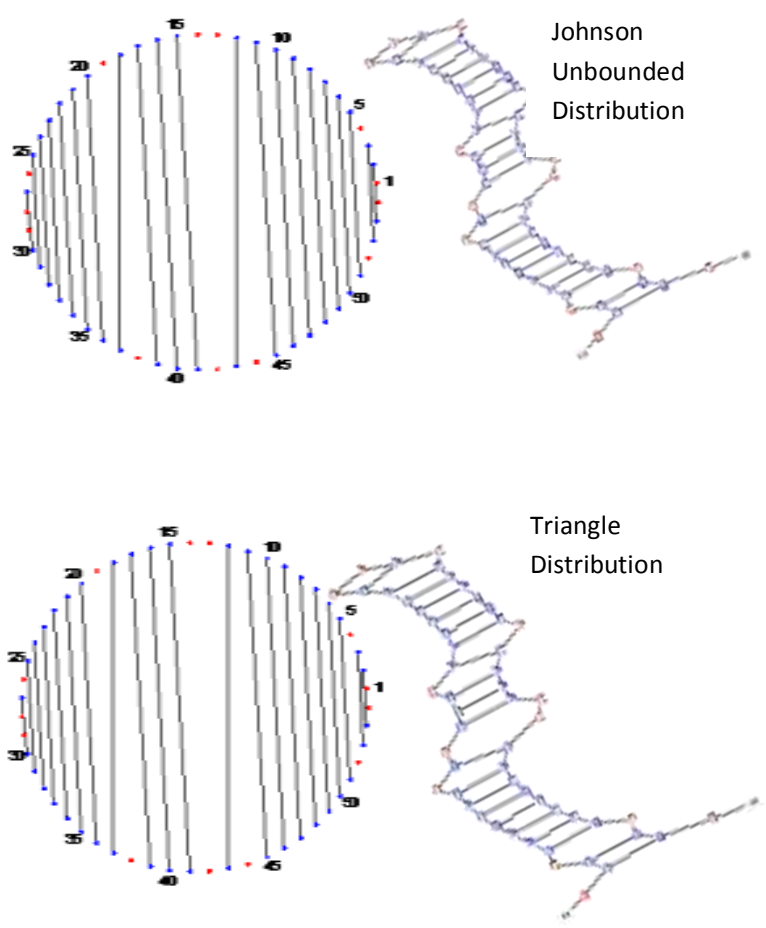

Figure 6 Proposed Secondary structures by all distributions

We execute the algorithm for all distributions mentioned above and this secondary structure is predicted by most of the distribution techniques applied here. Samples of other secondary structures predicted by the system for the same RNA sequence is shown in fig 6.

On the basis of frequency of hit on each base-pair and by mathematical inference, we deduce an optimal secondary structure from the proposed structures of every distribution and calculate its accuracy, deletion rate and false rate. The equations we used to calculate these measures are as follows:

$$
\begin{gathered}
\text { Accuracy }=\left(\frac{T P+T N}{T P+T N+F P+F N}\right) \\
\text { Deletion Rate }=\left(\frac{T P}{T P+F N}\right) \\
\text { False Rate }(\%)=\left(\frac{F P}{F P+T N}\right) \times 100
\end{gathered}
$$

Where, TP = True Positive, Base pairs that are predicted and exists in the known structure

$\mathrm{TN}=$ True Negative, Base pairs that are predicted but do not exist in the known structure

FP $=$ False Positive, Base pairs that are not predicted but exist in the known structure 
FN = False Negative, Base pairs that are neither predicted nor exist in the known structure

For these five distributions, table 2 shows the accuracy, deletion rate and false rate of the optimal structures found from proposed secondary structures of R17 viral RNA.

Table 2. Accuracy calculation of all the distributions

\begin{tabular}{|l|c|c|c|c|c|}
\hline & Uniform & Normal & JSB & JSU & Triangle \\
\hline TP & 3 & 14 & 19 & 19 & 19 \\
\hline FN & 15 & 9 & 3 & 3 & 3 \\
\hline FP & 17 & 6 & 1 & 1 & 1 \\
\hline TN & 323 & 328 & 335 & 335 & 335 \\
\hline Accuracy & 0.91 & 0.96 & 0.99 & 0.99 & 0.99 \\
\hline $\begin{array}{l}\text { Deletion } \\
\text { Rate }\end{array}$ & 0.17 & 0.61 & 0.86 & 0.86 & 0.86 \\
\hline $\begin{array}{l}\text { False } \\
\text { Rate }(\%)\end{array}$ & 5 & 1.8 & 0.3 & 0.3 & 0.3 \\
\hline
\end{tabular}

The results we obtained are same as predicted by Takefuji [25], but our machine learning algorithm predicted the structure in lesser time since the algorithm we used is quadratic in nature. Nevertheless our system finds out the optimal secondary structure by using statistical methods. There is no need to run the algorithm for long to converge to the solution. Our method finds out all possible secondary structures in just few hundred epochs but we intentionally run the algorithm for 8000 epochs. It was observed that machine learning method determines all possible secondary structures in first few hundred epochs and it repeat to predict the same secondary structures in remaining iterations.

Table 3. Impact of different distributions in the algorithm

\begin{tabular}{|r|r|r|r|r|r|}
\hline Seq & Uniform & Normal & \multicolumn{1}{c|}{ JSB } & \multicolumn{1}{c|}{ JSU } & Triangle \\
\hline 1 & 87.45 & 84.27 & 80.62 & 84.98 & 78.82 \\
\hline 2 & 15.81 & 65.28 & 90.58 & 90.58 & 90.58 \\
\hline 3 & 70.71 & 60.7 & 68.31 & 70.71 & 70.71 \\
\hline 4 & 83.21 & 78.45 & 83.21 & 83.21 & 83.21 \\
\hline 5 & 76.38 & 70.71 & 73.38 & 64.17 & 66.21 \\
\hline 6 & 59.63 & 81.41 & 83.91 & 83.91 & 62.62 \\
\hline & 65.53 & 73.46 & 80 & 79.59 & 75.35 \\
\hline
\end{tabular}

Table 3 shows the final result of our simulation. Results show the likelihood of reaching to stable structure using different distributions and it is evident from the table that Johnson Bounded and Unbounded distribution technique of random variable generation presents the most optimized results. It can be inferred that the transformation of normal distribution yields better results than the normal distribution itself.

\section{CONCLUSION}

We randomized the initial value of $U$ using different probability distributions and the results show that transformations of normal random variables works far better than any other technique of randomization. More specifically right skewed transformation yields the best result as compared to left skewed transformation of normal random variable. The system performed even better if the ranges of random variable vary between short intervals of negative values.

It is evident that working on base-pairs reduces much of the time and complexity in predicting the secondary structure of RNA. Time complexity may be reduced further by using parallel processing, like we present by using a very simple customized architecture of Neural Network. We use statistical techniques to obtain the most optimal secondary structure from the bulk of structures. This method reduces the time complexity by working on suboptimal structures rather than to let the system converge toward an optimal secondary structure, which may take a long time specifically with bigger sequences.

The algorithm we present here can further be modified by applying more thermodynamic rules and by taking nature and behavior of participating molecules into consideration.

\section{ACKNOWLEDGEMENT}

We pay special thanks to Miss Sidra Kazmi, who contributed with us in developing algorithms and for her worthy suggestions.

\section{REFERENCES}

[1] Eddy, S. R. (2001) Non-coding RNA genes and the modern RNA world, Nature Rev. Genet., 2, 919-929

[2] Storz, G. (2002) An expanding universe of noncoding RNAs, Science, 296, 1260-1263

[3] Schlick, T. (2002) Molecular Modeling and Simulation: An Interdisciplinary Guide, Springer-Verlag, New York, NY

[4] E. Westhof and P. Augginger, RNA Tertiary Structure, Encyclopedia of Analytical Chemistry, (C) John Wiley \& Songs Ltd.

[5] De Smit MH, van Duin J., Control of translation by mRNA secondary structure in Escherichia coli. A quantitative analysis of literature data. J.Mol Biol 1994, 244(2): 144-50

[6] De Smit MH, van Duin J., Secondary structure of the ribosome binding site determines translational efficiency: a quantitative analysis. Proceedings of the National Academy of Sciences 1990: 7668-7672

[7] Koev G, Liu S, Beckett R, Miller WA, The 3'-terminal structure required for replication of barley yellow dwarf virus RNA contains and embedded 3' end, Virology 2002, 292:114-126

[8] M. S. Waterman, Secondary structure of single-stranded nucleic acids, Studies in Foundations and Combinatories, Advan. In Math. Suppl. Studies, Vol 1, 167-212, Academic Press, New York, 1978 
[9] M. S. Waterman, Efficient sequence alignment algorithms, J, Theoret. Biol. 108 (1984), 333-337

[10] R. Nussinov, G. Pieczenik, J. R. Griggs, And D. J. Kleitman, Algorithms for loop matchings, SIAM J. Appl. Math. 35 (1978), 68-82

[11] Sankoff, D. (1985) Simultaneous solution of the RNA folding, alignment and protosequence problems, SIAM J. Appl. Math., 45, 810-825

[12] Zuker, M. 1989a "On finding all suboptimal folding of an RNA moleculr, Science, 244, 48-52

[13] Hofacker, I. L., Fontanna, W. Stadler, P.F., Bonhoffer, S., Tacker, M., and Schuster, P. 1994. Fast folding and comparison of RNA secondary structures (the Vienna RNA Package). Monath. Chem. 125: 167-188

[14] Zuker, M. and Stiegler, P. (1981). Optimal computer folding of large RNA sequences using thermodynamics and auxiliary information, Nucleic Acids Res. 9(1): 133-148

[15] Zuker, M. and Sankoff, D. (1984). RNA secondary structures and their prediction, Bull. Math. Biol. 46: 591-621.

[16] Takefuji, Y, Chen L., Lee, K., Huffman J. Parallel Algorithms for finding a near-maximum independent set of a circle graph, IEEE Transactions Vol 1., 1990

[17] Qi Liu, Xiuzi Ye and Yin Zhang, "A Hopfield Neural Network Based Algorithm for RNA Secondary Structure Prediction', IMSCCS '06, IEEE.
[18] Evan W. Steeg, Neural Networks, Adaptive Optimization, and RNA Secondary Structure Prediction, 1989, Artificial Intelligence and Molecular Biology

[19] Averill M. Law, W. David Kelton, "Simulation, modeling and analysis", $3{ }^{\text {rd }}$ edition, Tata McGraw-Hill Edition, 2004, ISBN 0-07-058290-4

[20] Johnson, N.L., 1949, System of Frequency Curves Generated by Methods of Translations, Biometrika, 36,149-158

[21] Tahseen A. Jilani, S. M. Aqil Burney, Huda Yasin, An Automated System for Hepatitis-C Patients' Classification Using PCA Based ANN, unpublished paper.

[22] I. Tinoco, O. C. Uhlenbeck, and M. D. Levine, "Estimation of secondary structure in ribonucleic acids", Nature vol. 230, 1971.

[23] Aqil Burney S. M., Jilani T. A., Cemal Ardil (2004), "Levenberg - Marquardt Algorithm for Karachi Stock Exchange Share Rates Forecasting", Published in International Journal of Computational Intelligence (IJCI), vol. 1(2), 2oo4. ISSN: 1304-4508, ISSN: 1304-2386, pp. $168-173$

[24] Aqil Burney S.M, Jilani T. A., Cemal Ardil (2004), “A Comparison of First and Second Order Learning algorithms in Artificial Neural Networks", International Journal of Computational Intelligence, vol. 1(3) 2004, pp. 218-224. ISSN: 1304-4508, ISSN: 1304-2386.

[25] Takefuji, Y. 1992 Neural Network Parallel Computing. Kluwer Academic Publishers. 